\title{
Hipertensão porta na esquistossomose mansônica: repercussões do tratamento cirúrgico no perfil histomorfométrico da mucosa gástrica
}

\author{
Portal hypertension in mansonic schistosomiasis: repercussions of surgical \\ treatment on the histomorphometric profile of the gastric mucosa
}

\author{
Mario Ribeiro de Melo-Júnior ${ }^{1,2}$, José Luiz de Figueiredo³, Jorge Luiz Silva Araújo Filho², \\ Marcos Cezar Feitosa de Paula Machado ${ }^{2}$, Carlos Teixeira Brandt ${ }^{4}$ \\ e Nicodemos Teles de Pontes-Filho ${ }^{2,5}$
}

\begin{abstract}
RESUMO
Investigou-se, através da análise digital de imagens, as repercussões do tratamento cirúrgico para controle da hipertensão porta e seus efeitos na vasculatura gástrica de pacientes jovens portadores de esquistossomose mansônica. Foram incluídos no estudo pacientes no pré-operatório $(n=5)$ e grupos de pacientes submetidos à intervenção cirúrgica, em diferentes períodos (0-2 anos, $n=04 ; 2-6$ anos, $n=13$; acima de 6 anos, $n=10$ ). Foram obtidas biópsias endoscópicas da mucosa do antro e corpo gástrico, submetidas à rotina histológica e montadas em blocos de parafina. Confeccionaram-se lâminas histológicas que foram usadas para a análise histomorfométrica dos seguintes parâmetros: número médio de vasos por campo, diâmetro médio e espessura da parede dos vasos. Os resultados obtidos evidenciaram uma diminuição significativa da densidade e do diâmetro dos vasos a partir dos dois anos de pós-operatório até o período superior a 6 anos. Os dados dão suporte ao conceito de que a técnica cirúrgica ministrada ameniza, em longo prazo, as alterações histopatológicas específicas, como a hemorragia e a ectasia.
\end{abstract}

Palavras-chaves: Gastropatia. Hipertensão porta. Esquistossomose mansoni. Morfometria.

\begin{abstract}
The repercussions from surgical treatment for controlling portal hypertension and its effects on the gastric vasculature of young patients with mansonic schistosomiasis were investigated by digital image analysis. The study included five patients at the preoperative stage and 27 patients who had undergone surgical intervention at different times in the past: 0-2 years ago, $n=4 ; 2-6$ years ago, $n=13$, and more than 6 years ago, $n=10$. Endoscopic biopsies were taken from the mucosa of the gastric antrum and body endoscopic mucosa and the samples underwent routine histological tests after embedding in paraffin blocks. Histological thin sections were used for histomorphometric analysis of the following parameters: mean number of vessels per field, and mean diameter and thickness of the vessel walls. The results showed that, between the patients whose operation was not more than two years ago and those whose operation was more than six years ago, there was a significant decrease in the density and diameter of the vessels. These findings give support to the concept that the surgical treatment administered decreases specific histological alterations like hemorrhage and ectasia, over the long term.
\end{abstract}

Key-words: Gastropathy. Portal hypertension. Schistosomiasis mansoni. Morphometry.

\footnotetext{
1. Disciplina de Patologia, Associação Caruaruense de Ensino Superior, Caruaru, PE. 2. Laboratório de Imunopatologia Keizo Asami, Recife, PE. 3. Departamento de Cirurgia, Centro de Ciências da Saúde, Universidade Federal de Pernambuco, Recife, PE. 4. Disciplina de Cirurgia pediátrica, Universidade Federal de Pernambuco, Recife, PE. 5. Departamento de Biologia, Universidade Federal Rural de Pernambuco, Recife, PE.

Endereço para correspondência: Prof. Mario Ribeiro de Melo Junior. Laboratório de Imunopatologia Keizo Asami/UFPE. Av. Prof. Morais Rego s/n, Campus Universitário. 50670-910 Recife, PE, Brasil.

e-mail: mariormj@gmail.com

Tel: $55812101-2504$

Recebido para publicação em 20/2/2006

Aceito em 19/1/2007
} 
A esquistossomose mansônica (EM) é uma helmintíase do sistema vascular porta mesentérico, provocada pelo Schistosoma mansoni ${ }^{6}$ que apesar de estar apresentando uma redução da prevalência na maioria das áreas endêmicas e diminuição das taxas de morbidade ainda constitui um problema de saúde publica no Brasil ${ }^{16}$.

A forma hepatoesplênica da EM conseqüente ao bloqueio do fluxo portal intra-hepático, provocado pelos ovos e granulomas que levam a uma fibrose em torno dos ramos portais (Fibrose de Symmers) $)^{2}$ e ao hiperfluxo esplênico secundário a esplenomegalia ${ }^{10}$, é expressada clinicamente pelo aumento do fígado e do baço. A hipertensão porta resultante desse fenômeno acomete 2 a $7 \%$ da população infectada no nordeste do Brasil e é a principal causa de hemorragia digestiva ${ }^{4}$.

Durante acompanhamento pré e pós-operatório das varizes esofagianas e da gastropatia dos pacientes com esquistossomose na forma hepatoesplênica, constatou-se que: em pacientes com hipertensão porta além das varizes esofagianas e do estômago, há uma ectasia vascular na mucosa gástrica, que foi descrita como gastropatia hipertensiva porta (GHP) ${ }^{12}$. Porém, algumas vezes, esta alteração tem sido confundida com a gastrite leve, sendo então necessária à realização de biópsias. Entretanto, muitos aspectos histopatológico da GHP ainda não estão totalmente esclarecidos ${ }^{25}$.

A fim de fornecer dados mais precisos, para estabelecer padrões histológicos confiáveis e específicos para as diversas doenças, várias técnicas morfométricas foram elaboradas durante as últimas décadas ${ }^{24}$, no intuito de introduzir na prática da análise histopatológica critérios objetivos e reproduzíveis concernentes ao diagnóstico e ao prognóstico de doenças ${ }^{17}$.

A identificação das alterações vasculares na mucosa do estômago dos pacientes com esquistossomose pode ser de grande significado não só para o diagnóstico da gastropatia como também para o controle da sua evolução, pois estas lesões podem ser causas de anemia, hipoproteinemia e sangramento gastrointestinal, contribuindo assim para o aumento da morbidade da doença ${ }^{1}$.

Investigando-se os processos inflamatórios gastrointestinais, tanto de etiologia parasitária ou não, utilizando-se da análise computadorizada de imagens, se obteve novas luzes para o melhor entendimento das alterações morfológicas no esôfago, estômago e intestino. Como por exemplo, na análise histomorfométrica da gastropatia congestiva ${ }^{7}$ e avaliação da mucosa gástrica em pacientes com hipertensão porta ${ }^{18}$.

Devido à escassez de estudos que elucidem essas questões a respeito da frequiência e tipo de alterações vasculares da gastropatia hipertensiva porta, o presente estudo avaliou 0 impacto da esplenectomia, ligadura da veia gástrica esquerda na microvasculatura gástrica de portadores jovens de esquistossomose mansônica hepatoesplênica através da análise histomorfométrica.

\section{MATERIAL E MÉTODOS}

Aspectos éticos. 0 delineamento experimental foi aprovado pelo Comitê de Ética em Pesquisa em Seres Humanos do Centro de Ciências da Saúde da UFPE em 10 de julho de 2002 conforme 0 ofício $n^{\circ}$ 204/2002-CEP/CCS. Todos os pacientes foram informados sobre a pesquisa e somente aqueles que concordaram em participar assinaram o termo de consentimento livre e esclarecido.

Casuística. Foram selecionados 33 portadores de esquistossomose mansônica, na forma hepatoesplênica, com sangramento digestivo alto que foram tratados clinicamente com Oxaminiquine na dose única de $20 \mathrm{mg} / \mathrm{Kg}$ (máxima de $600 \mathrm{mg} /$ dia) e após 30 dias, submetidos a esplenectomia, ligadura da veia gástrica esquerda e auto-implante esplênico no omento maior, no Serviço de Cirurgia Geral da Criança, do Hospital das Clínicas (HC) da UFPE, de 1990 a 2000.

Critérios de inclusão. Idades entre 7 e 15 anos quando submetidos ao tratamento cirúrgico; Idade media de 17 anos (variando de 12-22 anos) quando da realização da endoscopia; diagnóstico confirmado de Esquistossomose na forma hepatoesplênica; portadores de varizes do esôfago e hipodesenvolvimento ou hiperesplenismo; acometidos por fibrose de Symmers na análise histológica em biópsia do lobo esquerdo do fígado; submetidos a esplenectomia, ligadura da veia gástrica esquerda e auto-implante de tecido esplênico, em bolsa, no omento maior.

Critérios de exclusão. Antigenemia positiva para hepatite; alterações histológicas de cirrose hepática; patologias associadas como: cardiopatias nefropatias, AIDS e portadores de condições que possam influir na avaliação da mucosa gástrica como alcoolismo, doenças ulcerosas pépticas ou corticoterapia, uso de anti-inflamatórios não-hormonais.

Grupos experimentais. Após a triagem do pacientes (17 homens e 16 mulheres), os mesmos foram separados nos seguintes grupos: Pré - Grupo pré-operatório $(n=5)$; 0-2a-Grupo submetido à cirurgia com seguimento até dois anos $(\mathrm{n}=5) ; 2-6 \mathrm{a}-$ Grupo submetido à cirurgia com seguimento entre dois a seis anos $(n=13) ;>6 a-$ Grupo submetido à cirurgia com seguimento superior a seis anos $(n=10)$.

Estudos clínico-laboratoriais. Os exames endoscópicos foram realizados no setor de pesquisas clínicas do Laboratório de Imunopatologia Keiso Asami (LIKA) no Hospital das Clínicas - Universidade federal de Pernambuco, e no Serviço de vídeoendoscopia do Instituto Materno-infantil de Pernambuco (IMIP). Foram utilizados os vídeo-endoscópios modelo PENTAX EPM-3300 e OLIMPUS EVIS cv 1.000 .

Durante o ato endoscópico, realizamos biopsias da mucosa gástrica, obtendo dois fragmentos do antro e dois do corpo gástrico. Os fragmentos de mucosa gástrica de cada paciente, medindo aproximadamente $0,3 \times 0,3 \times 0,3 \mathrm{~cm}$, foram fixados em formalina a 10\%, submetidos à rotina histológica e incluídos em parafina. De todos as amostras foram obtidos cortes com $4 \mu \mathrm{m}$ de espessura, montados em lâminas histológicas em seguida coradas pela hematoxilina-eosina para análise histomorfométrica. As lâminas foram analisadas por um patologista confirmando a hipótese clínica e endoscópica.

Análise morfométrica e estatística. Para análise histomorfométrica foi utilizada a estação de Análise de imagens 
(sistema de vídeo-câmera acoplado a um microscópio óptico Olympus BH-2), já utilizado com sucesso em análises diagnósticas 9 . 0 sistema interativo de análise de imagens utiliza 0 Software OPTIMAS ${ }^{\circledR} 6.1$ e Câmera digital CCBBW 410 (Sansung), disponíveis no Departamento de Patologia da UFPE.

Os parâmetros morfométricos adotados foram à densidade média $\left(\right.$ vasos $/$ campo $\left.=12.234 \mu \mathrm{m}^{2}\right)$, diâmetro $(6$ campos aleatórios por lâmina) e espessura média dos vasos (3 vasos por lâmina histológica). Os valores obtidos foram analisados estatisticamente utilizando-se os testes de Tukey e teste t de Student. Aceitou-se $\mathrm{p}<0,05$ para retificação da hipótese de nulidade.

\section{RESULTADOS}

Estudo histopatológico. No estudo histológico das amostras de mucosa gástrica, não se evidenciaram diferenças significativas, tanto no aspecto qualitativo quanto no quantitativo, em nenhum dos parâmetros analisados entre as amostras do antro e do corpo gástrico.

Observou-se um nítido padrão de infiltrado inflamatório agudo no grupo pré-operatório e nos pacientes que se submeteram à cirurgia num período de tempo de até 2 anos. Por outro lado, a partir dos 2 anos até período superior a 6 anos da realização da cirurgia, a maioria (97,6\%) dos pacientes, apresentou padrão histológico compatível com o quadro de infiltrado inflamatório crônico, principalmente pela presença de células linfo-monocitárias e fibrose intersticial.

Quanto às alterações vasculares específicas, constatou-se que tanto a ectasia e hiperemia são achados histológicos que continuam presentes desde o período pré-operatório até um período superior a seis anos da realização da cirurgia. Por outro lado, a ocorrência de hemorragia nas amostras, apenas estava presente nos pacientes durante a fase pré-operatória (Tabela 1).

Tabela 1 - Parâmetros histológicos da gastropatia de pacientes jovens com hipertensão portal de etiologia esquistossomótica.

\begin{tabular}{lcccccc}
\hline & \multicolumn{5}{c}{ Parâmetro histológico** } \\
\cline { 2 - 7 } Grupo* $^{* *}$ & Infiltrado & Ectasia & Hiperemia & Hemorragia H. pylori Diagnóstico \\
\hline Pré & Agudo & +++ & ++ & ++ & ++ & GHPA \\
$0-2$ & Agudo & +++ & ++ & - & ++ & GHPL \\
$2-6$ & Crônico & ++ & ++ & - & + & GHPL \\
$>6$ & Crônico & - & - & - & + & GHPI \\
\hline
\end{tabular}

*Grupos: Pré: Pré-operatório, Pós-operatório (intervalo em anos): 0-2; 2-6; > 6 anos. **-ausente; + fraca; ++ moderada; +++ intensa.

GHPA: gastropatia hipertensiva portal ativa; GHPL: gastropatia hipertensiva portal leve; GHPI: gastropatia hipertensiva portal inativa

Análise histomorfométrica. Análise digital comparativa avaliou a densidade média dos vasos sanguíneos na região do antro e corpo gástrico, observando diferenças estatisticamente significativas entre o grupo pré-operatório e os demais grupos pós-operatórios $(\mathrm{p}<0,001)$, exceto para grupo que foi submetido à cirurgia a menos de dois anos (Figura 1).

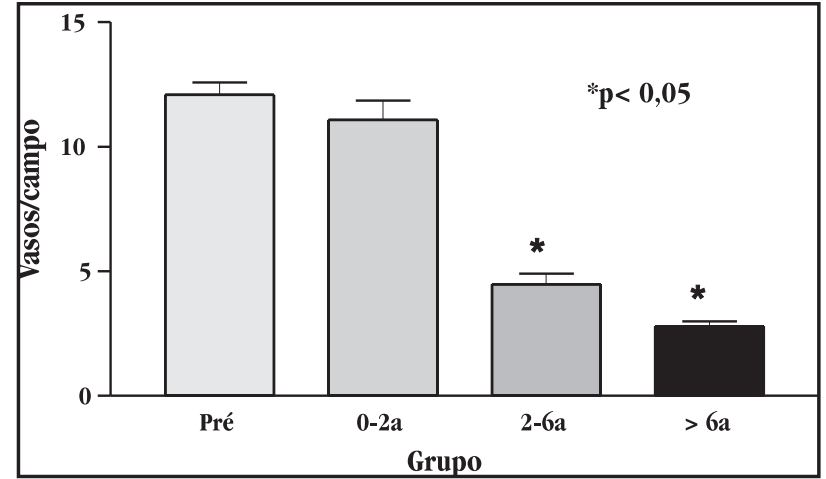

Figura 1 - Densidade média dos vazos sanguíneos da mucosa gástrica de pacientes jovens com esquistossomose mansônica submetidos a tratamento clínico e cirúrgico da hipertensão porta $\left({ }^{*} p<0,05\right)$.

Outro parâmetro histológico analisado foi o diâmetro médio dos vasos arteriais que revelou uma diminuição expressiva do diâmetro nos grupos pós-operatórios (2-6; > 6 anos) quando comparados ao grupo pré-operatório e pós-operatório, com menos de 2 anos da intervenção cirúrgica (Figura 2).

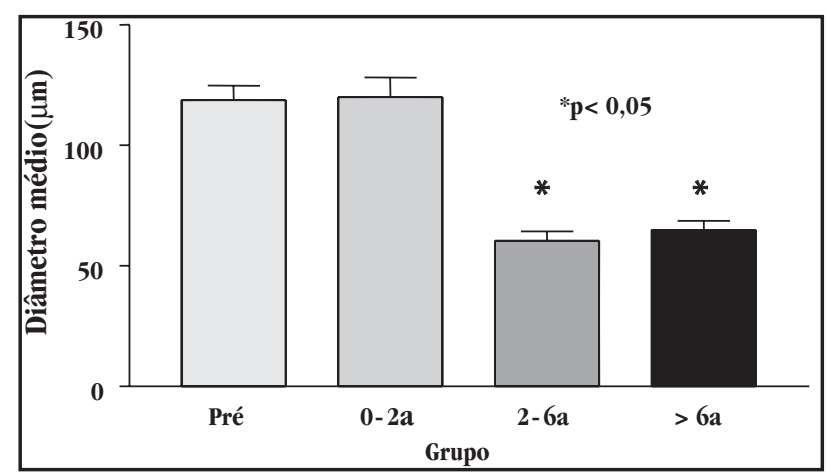

Figura 2 - Diâmetro médio dos vasos sanguíneos da mucosa gástrica de pacientes jovens com esquistossomose mansônica submetidos a tratamento da hipertensão porta.

A análise morfométrica da espessura média da parede dos vasos arteriais não evidenciou diferenças estatisticamente significativas quando se compararam os grupos de pacientes estudados (Figura 3).

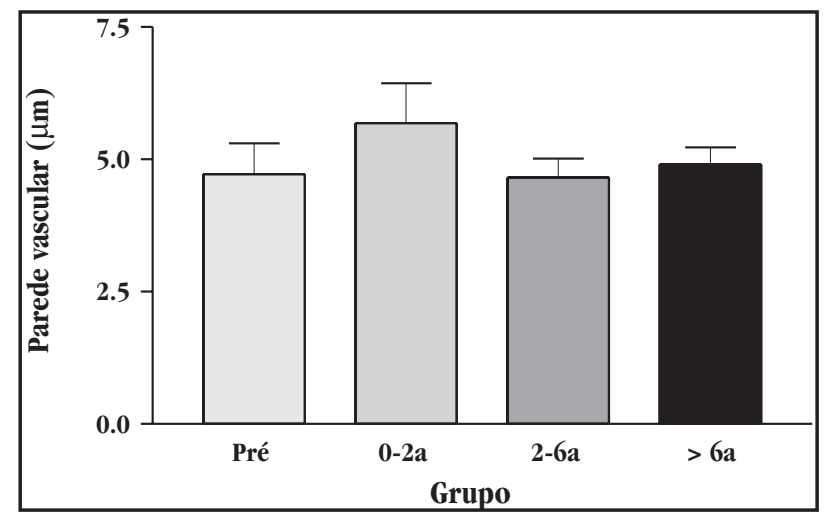

Figura 3 - Espessura média da parede dos vasos da mucosa gástrica de pacientes jovens com esquistossomose mansônica submetidos a tratamento clínico da hipertensão porta. 


\section{DISCUSSÃO}

0 presente estudo limitou-se à realização da endoscopia digestiva alta com biopsias do antro e corpo gástrico seguido da analise histomorfométrica da vasculatura, num grupo de pacientes jovens com a idade media de 17 anos (variando de 12-22 anos) quando da realização da endoscopia. Trata-se de uma abordagem nova devido ao fato de existirem poucos estudos histomorfométricos da gastropatia hipertensiva portal (GHP). Não existindo estudo similar em pacientes jovens com esquistossomose mansônica na forma hepatoesplênica.

O estudo dos distúrbios vasculares do esôfago e do estômago constitui um bom modelo para avaliação dos procedimentos terapêuticos clínicos e cirúrgicos, já que essas manifestações têm sido apontadas como achados mais relevantes da esquistossomose mansônica na forma hepatoesplênica ${ }^{19}$.

Considerando que a esplenectomia e a ligadura da veia gástrica esquerda é uma cirurgia que objetiva a redução do fluxo sanguíneo porta e conseqüientemente atenuação dos fenômenos de dilatação vascular na mucosa e submucosa do esôfago e do estômago, faz-se necessário o entendimento da dinâmica vascular porto-hepática dessa doença. Principalmente, quanto ao seu principal distúrbio, a hipertensão porta.

0 objetivo final do tratamento da hipertensão porta em pacientes portadores de esquistossomose mansônica na forma hepatoesplênica é prevenir a recidiva hemorrágica eliminando as varizes esofagogástricas, ou, pelo menos reduzir o calibre e a pressão das mesmas ${ }^{22}$. A hemorragia digestiva pode ocorrer em 12 a $20 \%$ dos pacientes, ocorrendo geralmente naqueles cuja pressão nas varizes esofágicas supera o nível de $20 \mathrm{mmHg}$. A recorrência, no entanto, é freqüente e imprevisível. Desta forma, o tratamento cirúrgico visa evitar a recidiva, procurando manter o equilíbrio hemodinâmico-funcional hepático característico da doença $\mathrm{a}^{19}$.

Utilizando-se de diferentes técnicas, vários grupos vêm focalizando suas pesquisas no entendimento das alterações hemodinâmicas envolvidas na hipertensão porta esquistossomótica, sugerindo que antes de se indicar qualquer tratamento cirúrgico deveria se determinar às alterações hemodinâmicas de cada caso. Seguindo esta mesma linha, um estudo recente ${ }^{15}$ demonstrou as alterações angiográficas, pressóricas, ultrassonográficas e dopplerfluxométricas determinadas pela esplenectomia e ligadura da veia gástrica esquerda na esquistossomose mansônica.

Alguns estudos dentre os quais destacam-se o de Geraghty ${ }^{8}$, e Casadewall ${ }^{5}$, demonstram que o período agudo após ligadura da veia porta é associado com a redução da microcirculação da mucosa do corpo gástrico más o efeito não é mantido nos intervalos tardios após a ligadura da veia porta, ocorrendo 0 aumento do fluxo sanguíneo mucoso gástrico, através da abertura de shunt portosistêmico.

Considerando que a fisiopatologia da gastropatia da hipertensão porta não está completamente esclarecida, uma vez que nem todos os pacientes com hipertensão porta desenvolvem alterações da mucosa gástrica, é possível que outros fatores estejam implicados na patogênese da GHP ou então o método de investigação necessita ser melhor adequado.
Sabendo-se que dilatação é definida como uma expansão do lúmen vascular sem alteração na estrutura do vaso, enquanto que ectasia é um alargamento do lúmen por modificação estrutural da parede do vaso; e considerando que os termos dilatação e ectasia são muitas vezes utilizados como sinônimos e que alguns autores têm se referido a GHP apenas como significante dilatação vascular ${ }^{18} \mathrm{e}$ outros como um espessamento na parede de um vaso dilatado ${ }^{23}$. Desta forma, se faz necessário empreender mais estudos a fim de estabelecer critérios mais precisos na avaliação destes parâmetros.

Neste trabalho, de acordo com os resultados da avaliação histomorfométrica, pôde-se constatar que a cirurgia de esplenectomia e ligadura da veia gástrica esquerda, parece ter contribuído para uma redução dos episódios de hemorragia gástrica, devido ao fato de não terem sido encontradas novas ocorrências no grupo pós-operatório. 0 que de certa forma sugere uma redução da pressão nos vasos gástricos.

Estudos, através de cintilografia, observaram proeminentes fístulas artério-venosas na microcirculacão da mucosa gástrica em indivíduos com hipertensão porta ${ }^{21}$, esses achados podem ser conseqüentes a um aumento da pressão sangïínea na vasculatura gástrica, o que poderia justificar os resultados do presente estudo quanto ao aumento da densidade e diâmetro dos vasos da mucosa gástrica nos pacientes do grupo pré-operatório, e uma diminuição da densidade e do diâmetro desses vasos, possivelmente conseqüente diminuição do hiperfluxo, num período acima de dois anos após o tratamento cirúrgico.

É sabido que a hipertensão porta leva a distúrbios hemodinâmicos no trato gastrintestinal (TGI). Detalhados estudos histomorfométricos de biopsias do trato TGI (estômago, duodeno e jejuno) têm revelado significativas mudanças nos vasos da mucosa (veias e capilares) ${ }^{13}$. Desta forma, buscou-se neste trabalho, utilizar métodos quantitativos para estabelecer padrões mais precisos e significativos do perfil vascular da GHP no período pré-operatório e em três intervalos do pós-operatório de um grupo de pacientes jovens com esquistossomose mansônica na forma hepatoesplênica.

Uma abordagem semelhante também foi feita em estudo anterior $^{20}$, que também utilizou métodos morfométricos para medir o número e diâmetro dos vasos entre pacientes com hipertensão porta ocasionada pela cirrose alcoólica, e concluíram que a utilização desses métodos são eficazes para o estudo das anormalidades vasculares da mucosa gástrica. Fato similar também foi relatado na colopatia da hipertensão porta ${ }^{14}$.

Trabalho usando análise morfométrica de biópsias da mucosa gástrica encontrou um diâmetro capilar médio significativamente maior nos pacientes cirróticos quando comparado ao grupo controle $^{18}$. Esses resultados também foram encontrados no presente estudo nos pacientes do grupo pré-operatório.

Outros estudos ${ }^{311}$, investigando biopsias do antro e corpo gástrico de pacientes com GHP constataram a ocorrência de vasos dilatados e tortuosos com paredes espessadas. Fato este, não totalmente encontrado nos presentes resultados, pois não se observam diferenças estatisticamente significantes na espessura média dos vasos tanto nos pacientes do grupo pré quanto do pós-operatório. 
Os achados morfométricos do presente trabalho que indicam diminuição da densidade e diâmetro médio dos vasos da mucosa gástrica reforçam a idéia de que o tratamento cirúrgico empregado deve favorecer um menor estresse causado pela hipertensão, induzindo assim, um equilíbrio da trama vascular nesta região. Principalmente, nos indivíduos submetidos à cirurgia num período superior a dois anos.

Em conclusão, o estudo histomorfométrico das repercussões da esplenectomia e ligadura da veia gástrica esquerda, na microvasculatura gástrica de portadores jovens de esquistossomose mansônica hepatoesplênica, determina: 1) Redução significante na densidade e diâmetro médio dos vasos da mucosa gástrica em pacientes submetidos à cirurgia num período mínimo de dois anos e superior a seis anos; 2) Não houve diferença estatisticamente significante na espessura da parede dos vasos da mucosa gástrica de pacientes jovens com esquistossomose mansônica entre o préoperatório e os diferentes períodos de pós-operatório. 3) A partir de parâmetros histológicos como: tipo de infiltrado inflamatório, ectasia, hiperemia e hemorragia, podem-se constatar padrões distintos para a gastropatia hipertensiva portal ativa (GHPA); gastropatia hipertensiva portal leve (GHPL) e gastropatia hipertensiva portal inativa (GHPI).

\section{REFERÊNCIAS}

1. Bica I, Hamer DH, Stadecker MJ. Hepatic schistosomiasis. Infectctions Disease Clinnical North Americana 14: 583-604, 2000.

2. Bogliolo L. Subsídios para o conhecimento da forma hepato-esplênica da forma toxêmica da esquistossomose. Serviço Nacional de Educação Sanitária, Ministério da Saúde, Rio de Janeiro, 1958.

3. Brandt CT, Figueiredo JL, Almeida S, Guendler AV, Jucá N, Mendes S. Esquistossomose hepatoesplênica em jovens submetidos a esplenectomia e ligadura da veia gástrica esquerda. Estudo prospectivo das varizes esofagianas. Anais da Faculdade de Medicina da Universidade Federal de Pernambuco 47:14$17,2002$.

4. Carmo EH, Bina JC, Barreto ML. Schistosomiasis related morbidity in Brazil; Spatial distribution, clinical features evolution and medical services assessment. In: Simpósio internacional sobre esquistossomose, Recife-PE 6:166, 1997.

5. Casadewall M. Time course changes in gastric mucosal blood perfusion following portal vein constriction in rats. Journal of Hepatology 23:193-198, 1995

6. Domingues ALC, Barreto VST. Esquistossomose hepática. In: Matos AA, Dantas W. Conferência de Hepatologia. Fundo Editorial Byk, São Paulo: p.391-405, 2001.

7. Domingues ALC, Domingues LAW. Forma Intestinal hepatointestinal e hepatoesplênica. In: Malta J (ed) Esquistossomose mansônica. Editora Universitária da Universidade Federal de Pernambuco, Recife p.91-109, 1994.
8. Geraghty JG, Angerson W, Carter D. A study of regional gastric mucosal blood flow in a rat model of the hepatic cirrhosis. American Journal of Physiology 262:6727-6731, 1992 .

9. Hamilton PW. Interactive computer-aied morphometry. In: Peter W, Hamilton, Derek CA (eds) Quantitative clinical pathology. Blackwell-Sience, USA, 1997.

10. Lacerda CM. Esplenectomia e ligadura da veia gástrica esquerda na esquistossomose mansônica. Efeito sobre a pressão das varizes de esôfago aferida por técnica não invasiva. Tese professor Titular. Faculdade de Ciências Médicas de Pernambuco. Recife, PE, 1997.

11. Lamps ML, Hunt CM, Green A, Gray GF. Alterations in mucosal vessels in patients with cirrhosis and non-cirrhotic portal hypertension. Human Pathology 5: $527-$ $535,1998$.

12. McCormack TT, Simns J, Eyre-Book I, Kennedy H., Goepel J, Johnson AG, Triger DR. Gastric lesions in portal hypertension inflammatory gastritis or congestive gastropathy? Gut 26:1226-1232, 1985.

13. Misra SP, Dwivedi M, Misra V. Prevalence and factors influencing hemorrhoids, anorectal varices and colopathy in pacients with portal hypertension. Endoscopy 29:340-345, 1996.

14. Misra V, Misra SP, Dwivedi M. Thickened gastric mucosal capillary wall: a histological marker for portal hypertension. Pathology 30:10-13, 1998.

15. Pereira FMF. Alterações angiográficas, pressóricas, ultra-sonográficas, e dopplerfluxométricas determinadas pela esplenectomia e ligadura de veia gástrica esquerda na esquistossomose mansônica. Tese de Doutorado, Universidade Federal de Pernambuco, Recife, PE, 2003.

16. Pereira G, Santos RP, Alexandre Neto J, Azevedo AP, Carvalheira AE. Formas graves da esquistossomose mansônica: dados de internação hospitalar em Pernambuco. Anais da Faculdade de Medicina da Universidade Federal de Pernambuco 38: 12-18, 1993.

17. Petersen I, Wolf G, Roth K, Schlüns K. Telepathology by the internet. Journal Pathology 191: 8-14, 2000.

18. Quintero E, Pique JM, Bombi JA. Gastric mucosal vascular ectasias causing bleeding and cirrhosis. Gastroenterology 93:1054-1061, 1987.

19. Raia S, Mies S, Alfieri F. Portal hypertension in mansonic schistosomiasis. World Journal of Surgeny 15:176-187, 1991.

20. Sallebert S, Mancheron H, Sevestre H, Dupas JL, Capron JP. Histomorphometric study of the gastric mucosa in portal hipertension. Clinical Gastroenterology 12:89-92, 1988.

21. Sarfeh IJ, Soliman H, Waxman K, Coccia M, Rypins EB, Bui HX, Tarnawski A. Impaired oxygenation of gastric mucosa in portal hypertension. Digestive Diseases and Science 34:225-228, 1989.

22. Strauss E, Sakai P, Gayotto LCC, Foster S, Raia S. Size of gastroesohageal varices: its behavior after surgical treatment hypertension. Revista do Hospital das Clínicas de São Paulo 57: 1-8, 1999.

23. Tarnawski A, Sarfeh IJ, Stachura J. Microvascular abnormatlities of portal hypertensive gastric mucosa. Hepatology 8: 1488-1494, 1998.

24. True LD Morphometry applications in anatomic pathology. Human Pathology 27: 450-467, 1996.

24. Viggiano TR, Gostout C. Portal hypertensive intestinal vasculopathy: a review of the clinical, endoscopic, and histopathologic features. American Journal of Gastroenterology 87: 944-946, 1992. 